\title{
EMD Method Applied to Identification of Logging Sequence Strata
}

\author{
Ni ZHAO ${ }^{1,2}$ and Rui $\mathrm{LI}^{1}$ \\ ${ }^{1}$ State Key Laboratory of Oil and Gas Reservoir Geology and Exploitation (CDUT), \\ Chengdu, China \\ ${ }^{2}$ Xi' an Technological University, Computer Science and Engineering College, \\ Xi’an, China; e-mail: zhaoni369@126.com (corresponding author)
}

\begin{abstract}
In this work, we compare Fourier transform, wavelet transform, and empirical mode decomposition (EMD), and point out that EMD method decomposes complex signal into a series of component functions through curves of local mean value. Each of Intrinsic Mode Functions (IMFs component functions) contains all the information on the original signal. Therefore, it is more suitable for the interface identification of logging sequence strata.

Well logging data reflect rich geological information and belong to non-linear and non-stationary signals and EMD method can deal with non-stationary and non-linear signals very well. By selecting sensitive parameters combination that reflects the regional geological structure and lithology, the combined parameter can be decomposed through EMD method to study the correlation and the physical meaning of each intrinsic mode function. Meanwhile, it identifies the stratigraphy and cycle sequence perfectly and provides an effective signal treatment method for sequence interface.
\end{abstract}

Key words: empirical mode decomposition (EMD), intrinsic mode function (IMF), logging data processing, sequence stratigraphy.

Ownership: Institute of Geophysics, Polish Academy of Sciences;

(C) 2015 Zhao and Li. This is an open access article distributed under the Creative Commons Attribution-NonCommercial-NoDerivs license,

http://creativecommons.org/licenses/by-nc-nd/3.0/. 


\section{INTRODUCTION}

Sequence stratigraphy is a branch of stratigraphy, and it studies chronostratigraphic framework based on erosion surface or comparable integrated surface, inner stratum of sedimentary sequence, and petrographic distribution pattern which is in a cycle type in time and internally linked by formation. Sequence partition and interface identification are the foundation to study sequence stratigraphy. Generally, outcrop data, core data, logging data, and seismic data are adopted for the partition and identification of sequence stratigraphy. The logging data contains rich geologic information. When the information from outcrop and core is not enough and seismic resolution is limited, logging data becomes more important during the partition of sequence stratigraphy (Van Wagoner et al. 1990).

As the theory of high resolution sequence stratigraphy puts forward, various methods such as wavelet analysis, Hilbert-Huang transform (HHT) are applied into strata partition. Hilbert-Huang transform is an arithmetic method, based on Hilbert spectrum. It includes empirical mode decomposition and Hilbert Spectrum Analysis. Empirical mode decomposition, EMD method for short, calculates local mean curve for instable signals, which decomposes the complex signal to a series of component functions for instantaneous frequency with obvious physical meaning. This method is very useful to deal with non-stationary and non-linear processes (Huang et al. 1998, 1999; Wu and Huang 2004). In this study, the EMD method is applied into logging data processing. It serves as an effective method for identifying sequence interface in logging.

\section{EMPIRICAL MODE DECOMPOSITION (EMD) METHOD}

\subsection{Theoretical basis of EMD method}

EMD is a kind of transform method, in which analysis should be adaptable to the nature of the data. Its essential is to obtain signal's intrinsic fluctuating mode by using the characteristic temporal scale of signal (Li et al. 2010). Compared with wavelet transform and Fourier transform, the EMD transform is ideally suitable for handling data from non-stationary and non-linear processes. EMD method can decompose any complicated data into a small number of intrinsic mode function components, IMFs, which represent the basic characteristics of the data. As it is adaptable and the decomposition is based on the local characteristics of the data, the IMFs usually are monocomponent oscillatory modes. Specifically, this method is based on the following hypothesis:

(i) A signal shall have a maximum value and a minimum value;

(ii) The characteristic temporal scale shall be defined by the time interval of extreme points. 


\subsection{Decomposing procedure of EMD method}

Effective algorithm procedure of EMD of a signal $x(t)$ is presented in Fig. 1.

Intrinsic mode functions (IMFs) have to satisfy the following two conditions:

(i) in the whole data set, the number of extreme values and the number of zero crossings must be either equal or differ at most by one;

(ii) at any point the mean value of the envelope defined by the local maxima and the envelope defined by the local minima is zero (de Lima et al. 2006).

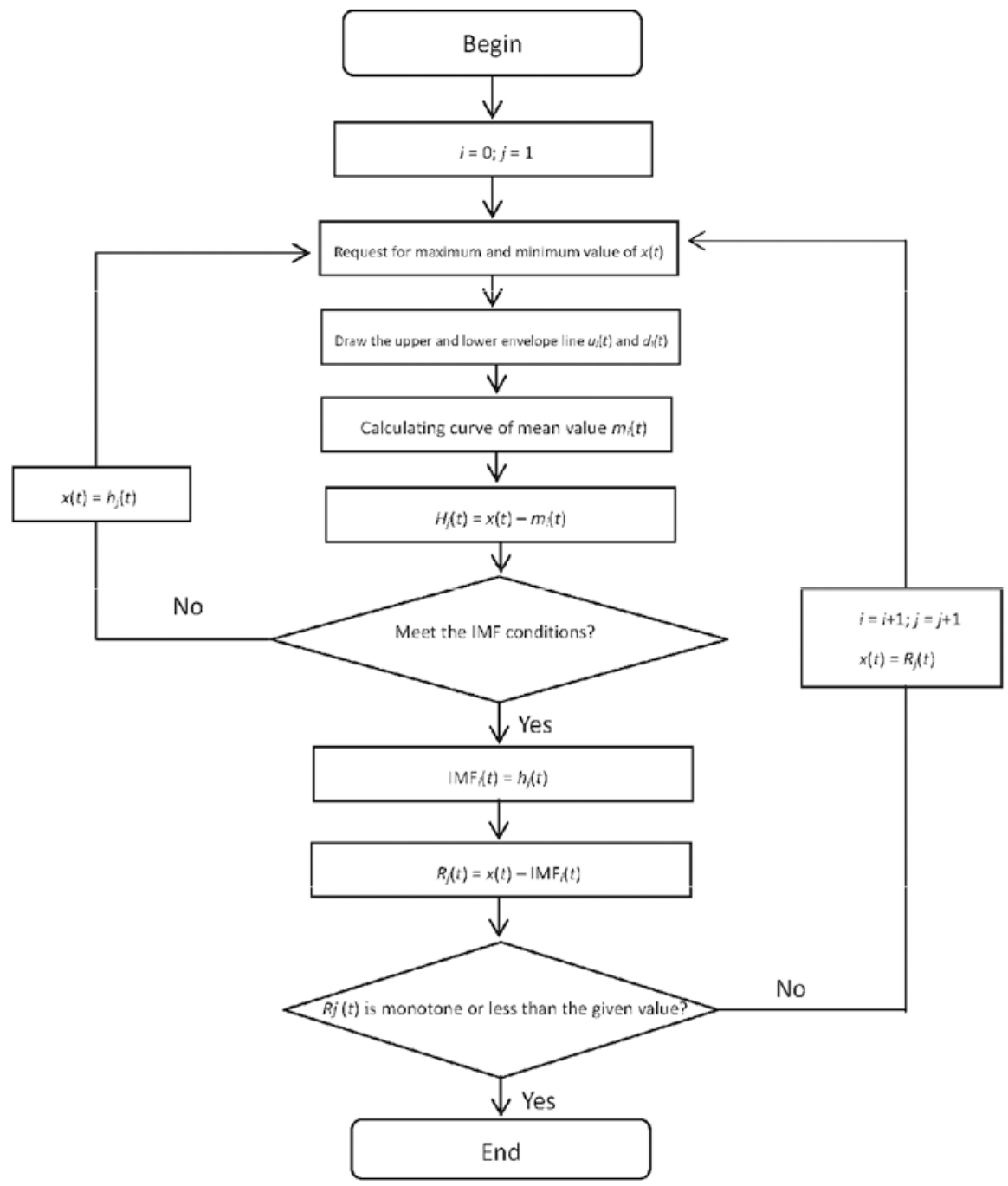

Fig. 1. Decomposing procedure of EMD. 
The first condition guarantees that the maximum value of datum of local part is positive and minimum value is negative; the second condition eliminates the instantaneous frequency vibration caused by nonsymmetrical waveform (Huang et al. 1998).

For a given signal $x(t)$, EMD ends up with a representation of the form:

$$
x(t)=\sum_{i=1}^{n} \operatorname{IMF}_{i}(t)+R(t) .
$$

In Eq. 1, $R(t)$ stands for a residual trend and the intrinsic mode functions $\left\{\mathrm{IMF}_{i}(t), i=1, \ldots n\right\}$ are the modulating wave of frequency-amplitude modulation constrained by zero-mean value (Flandrin et al. 2004).

As depicted in Fig. 1, when the first IMF has been calculated successfully, it is separated from the original signal and produced a residual. This residual is considered as a new signal to be computed again. It was repeated until the residual no longer contains any oscillations (Bowman and Lees 2013).

The process shows that the starting point of EMD is to consider signals at the level of their local oscillations. EMD method is used to decompose original signal into a series of intrinsic mode functions (IMFs) from high frequency to low frequency as well as a residual trend item, namely $\operatorname{IMF}_{i}(t)$ and $R(t)$, respectively. Once this decomposition (EMD) is achieved, details are considered as effective and consisting of all local feature.

In practice, the EMD sifting process was multi-scale, adaptable, and based solely on the data, so we can find an appropriate scale that may reveal important information embedded in the original signal.

\subsection{Advantages of EMD}

Fourier transform and wavelet analysis are the common methods in logging signal analysis. Now, EMD method is compared with them:

Fourier transform is the foundation for analyzing signal frequency spectrum. It decomposes an original signal into weighted sum of several sine signals, of which each sine signal aims at one fixed frequency and amplitude value. It is suitable for analyzing stable signal that does not change with time (Wu and Huang 2004). Logging data is instable signal-in-space. Through Fourier transform, frequency signal can be obtained. It has a maximum resolution in the frequency domain but it does not contain depth information, so you may find the maximum frequency but you cannot confirm its depth.

Wavelet transform is the extension of Fourier transform. It can adjust the size of window automatically according to the frequency (Wawrzyniak 2010). Meanwhile, it has more resolving and analytic functions. Discrete wavelet decomposition produces low frequency signal and high frequency signal generated from two complementary filters (Akansu et al. 2010). In 
application, low frequency part is regarded as the most important part. The limitation of wavelet transform includes: first, the selection of wavelet function shall influence the accuracy of decomposing, and secondly, each extraction does not contain the complete information. It only represents the part after filtering.

EMD method is the key part of HHT and it is designed specifically for adaptable representation of non-stationary and non-linear signal processes. It can be used to decompose any complicated data into sums of intrinsic mode functions plus a residual (Huang and $\mathrm{Wu}$ 2008). EMD provides us with a useful method to find out the underlying processes of non-stationary and non-linear signals and better indicate the physical meaning of local phase change with the instantaneous frequency than any other non-IMF time series. EMD decomposes the whole signal and each component contains whole information that can reflect the characteristics of instantaneous frequency (Zheng and Yang 2007). This is the major advantage of EMD method compared to wavelet transform.

\section{EMD METHOD APPLICATION IN IDENTIFICATION OF LOGGING SEQUENCE STRATA}

\subsection{Parameters selection and processing}

During well logging data collection, various interference factors cannot be avoided, which must influence the resolution of signal. Using a linear combination of sensitive parameters, highly correlated, is an effective method to improve accuracy of logging interpretation. At the beginning of processing well logging signals data should be deeply analyzed and various parameters should be compared. As a result, sensitive parameters reflecting lithological characteristics will be selected. Next, cross-correlation coefficients on the selected sensitive parameters such as spontaneous potential and natural gamma ray, and transit interval time and density, etc. will be calculated. Combining various linearly correlated parameters can strengthen stratum characteristics information. Because the noise is generated randomly, stacked signals with good correlation can highlight useful information and decrease the influence of interference factors.

In order to stack groups of data with different units, normalization should be applied and, next, sets of non-dimensional data can be used to form a new analytic parameter.

\subsection{Identification method of circle of basic level}

\subsubsection{Data processing rule}

EMD decomposes the original signal into intrinsic functions $\left(\mathrm{IMF}_{i}\right)$ with different frequency and a remaining trend item $R$. Each IMF is the mode of a 
signal at some characteristic dimension. The component with high frequency reflects the short-term cycle characteristics that is mainly controlled by astronomical factors (such as long eccentricity, precession cycle), while the component with low frequency reflects the middle and long term stable cycle characteristics that is controlled by long term structure formation process (such as regional tectonic movement, the tectonic of stress field transformation, and tectonic episodic intensity change). $R$ refers to the data trend or zero drift of instruments (Zhang and Nie 2011).

Based on this rule, correlation of intrinsic mode function $\mathrm{IMF}_{i}$ obtained after EMD can be determined as follows:

(i) calculate the correlation coefficient of every $\mathrm{IMF}_{i}$ and its original signal. Commonly, those IMFs which have good correlation with the original signal are the best signal components reflecting stable sedimentary environment;

(ii) analyze the correlation between different IMFs and combine those $\mathrm{IMF}_{i}$ with good correlation. This is because that good correlation reveals that they have consistent variation style. Combining them can reduce interference factors and strengthen signals that reflect the geological characteristics; therefore, it can improve the accuracy of bed boundary identification.

\subsubsection{EMD response characteristics}

After the well logging signal is decomposed using EMD method, the timefrequency characteristics of original well logging signals can be obtained from different dimensions, which can reflect different sedimentary cycle grades. If the correlation with all kinds of sequence interfaces can be identified, then it can be deemed as the standard to divide the sequence of well logging.

When the river reaches balance under the dynamics, suppose the balanced profile as a potential energy surface, called the base level. The land surface or sedimentary boundary will develop towards the base level through sedimentary or erosion action to reach a new balance. Such a circling process is called sequence formation cycle (Zheng et al. 2000, 2001). This kind of cycle of sequence stratum is reflected in well logging data as rhythmicity of mineral grains granularity and lithology. It is reflected as different amplitude and frequency characteristics in well logging curve. Yet, the break points of a curve are often the sequence interfaces (Serra and Abbott 1982). Based on this, it is useful to make the mirror image on response characteristics of EMD curve of well logging parameters reflecting all kinds of sedimentary environment and summarize five basic types (Table 1). Most well logging curves of different sedimentary environments are the combination or variants of these five basic types. 
Basic types of sedimentary environment that is corresponding to EMD curve of well logging

\begin{tabular}{|c|l|l|}
\hline Gradually- \\
varied type
\end{tabular}

\section{PRACTICAL EXAMPLE}

EDM method was applied for the $\mathrm{X}$ well logging data, Xujia River Group, middle section of West Sichuan Depression, Sichuan Basin. The sequence stratum was divided by using EMD and the cycle of base level was identified. 


\subsection{Analysis on parameter combination}

Natural gamma ray (GR) log is a common logging method to measure intensity of natural gamma ray in rock along the well bore. Rocks contain different amounts of radioactive elements and constantly emit radiation. GR curve reflects the granularity, sorting and clay minerals content, which can be used to judge lithology, compare stratum, and estimate the shale content. Commonly, gamma ray readings in sandstone and clay-stone are low and high, respectively. Yet, when sandstone has radioactive minerals such as mica or zircon or others, the reading of gamma ray will be influenced directly.

Spontaneous potential (SP) log is an effective logging method for analyzing geological profile in borehole, which can be used to measure shaft spontaneous potential changes in an open hole. SP curve is often used to divide the sandy-shaly profile into sandstone and shale lithology, compare the stratum, define the interface position of filtering layer, calculate the content of shale of the stratum, and spread the sedimentary face to study, etc.

The presented analysis indicated that these two well logging data (GR and SP) are the sensitive parameters that are suitable for sequence division of the study area. At the same time, their curves indication have consistent rule: at the profile of sandstone and shale section well, the value of these two curves is low at sandstone while the value is high at shale. So, these parameters can be combined.

Correlation coefficient between natural gamma ray and spontaneous potentials parameters was equal to 0.44 , which indicated that correlation was sufficient. Normalization treatment on natural gamma ray and spontaneous potentials curves was done to eliminate the influence of units and select linear normalization formula:

$$
y=\frac{x-\operatorname{Min}}{\operatorname{Max}-\operatorname{Min}} .
$$

In Eq. 2, $x$ refers to current parameter value, Max and Min are $x$ 's maximum and minimum value, respectively, and $y$ is the value after $x$ normalization.

With this expression the normalized values of GR, namely GR' (Eq. 3) and $\mathrm{SP}^{\prime}$ (Eq. 4), were calculated:

$$
\begin{aligned}
\mathrm{GR}^{\prime} & =\frac{\mathrm{GR}-\mathrm{GR}_{M i n}}{\mathrm{GR}_{M a x}-\mathrm{GR}_{\text {Min }}}, \\
\mathrm{SP}^{\prime} & =\frac{\mathrm{SP}-\mathrm{SP}_{\text {Min }}}{\mathrm{SP}_{M a x}-\mathrm{SP}_{M i n}}
\end{aligned}
$$


Mean values of the normalized parameters $\left(\mathrm{GR}^{\prime}\right.$ and $\left.\mathrm{SP}^{\prime}\right)$ were combined to form a new parameter marked GR_SP:

$$
\mathrm{GR} \_\mathrm{SP}=\frac{\mathrm{GR}^{\prime}+\mathrm{SP}^{\prime}}{2} \text {. }
$$

Analyzing the correlation of GR_SP and natural gamma ray (GR) and spontaneous potentials (SP), there was found the correlation coefficient of GR_SP and GR equal to 0.94, and the correlation coefficient of GR_SP and SP equal to 0.73 . This shows that the new parameter had higher correlation with natural gamma ray and spontaneous potentials. It can replace the original parameters.

\subsection{Cycle identification and stratigraphic division}

Following the EMD decomposition process presented in Fig. 1 a program with MATLAB was prepared. Adopting EMD on GR_SP data of X well, Xujia River Group, middle section of West Sichuan Depression, Sichuan Basin, the intrinsic mode functions IMF were obtained (Fig. 2).

IMF1-IMF4 reflected strong vibrations of the signal (Fig. 2). Yet, the positions of high amplitude value were different. This showed the influence of noise on high frequency component. As the frequency of all IMF signals declined, the stable transform trend in signal became stronger and stronger.

A different intrinsic mode function component can be obtained through the decomposition of EMD. Superposing the decomposed components, the original signal may be reproduced. Using such characteristics, we repeated the reverse accumulation of the intrinsic mode functions IMF1-IMF9 and trend item $R$ of each stages of GR_SP in Fig. 2, namely, the combination from the component with low frequency to high frequency (Fig. 3).

IMF9, IMF7, IMF6 or IMF5 acted importantly in original sequence (Fig. 3). The changes of original sequence were mainly caused by the oscillation of these four functions. They all had consistent change pattern. This pattern reflected the characteristics of stable sedimentary environment of the stratum.

Specifically, the wave scope of IMF5, IMF6, and IMF7 covered several meters to tens of meters and the wave width of IMF9 is over one hundred meters. The cycles with different base levels commonly are divided according to time distribution. In practical layers, the thickness of short-term cycle is of several meters to dozens of meters. The middle term cycle is dozens of meters to nearly one hundred meters and the long term cycles are nearly one hundred meters to several hundred meters. Therefore, IMF5, IMF6, and IMF7 reflects the short-term circle characteristics, while IMF9 the middle and long term characteristics. 

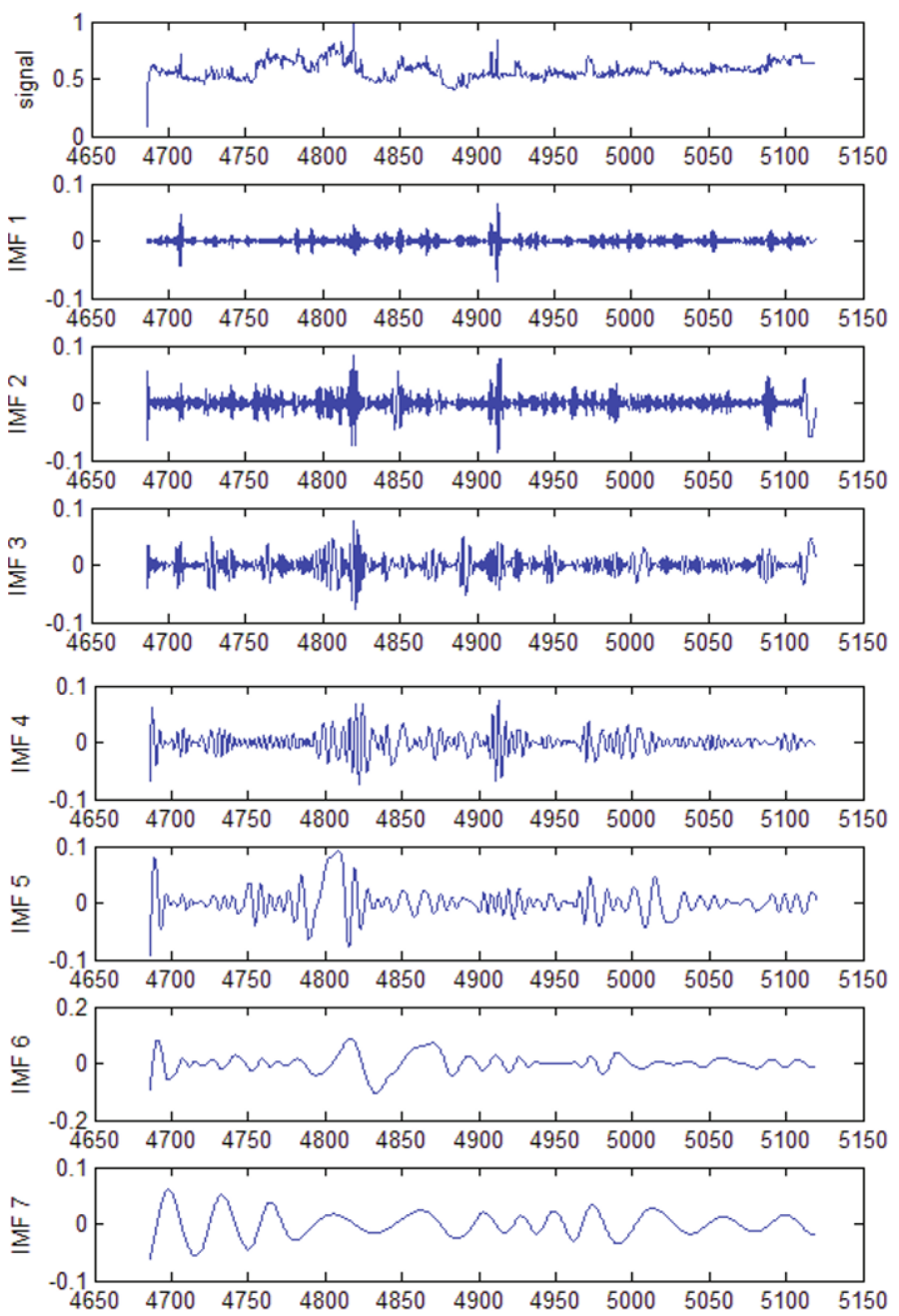

Fig. 2. EMD diagram of GR_SP (IMFs 1-9 are the empirical mode decompositions (EMD) of the signal which is the new combined parameter between GR and SP). $\quad R$ represents the final residual which showed the data trend. 

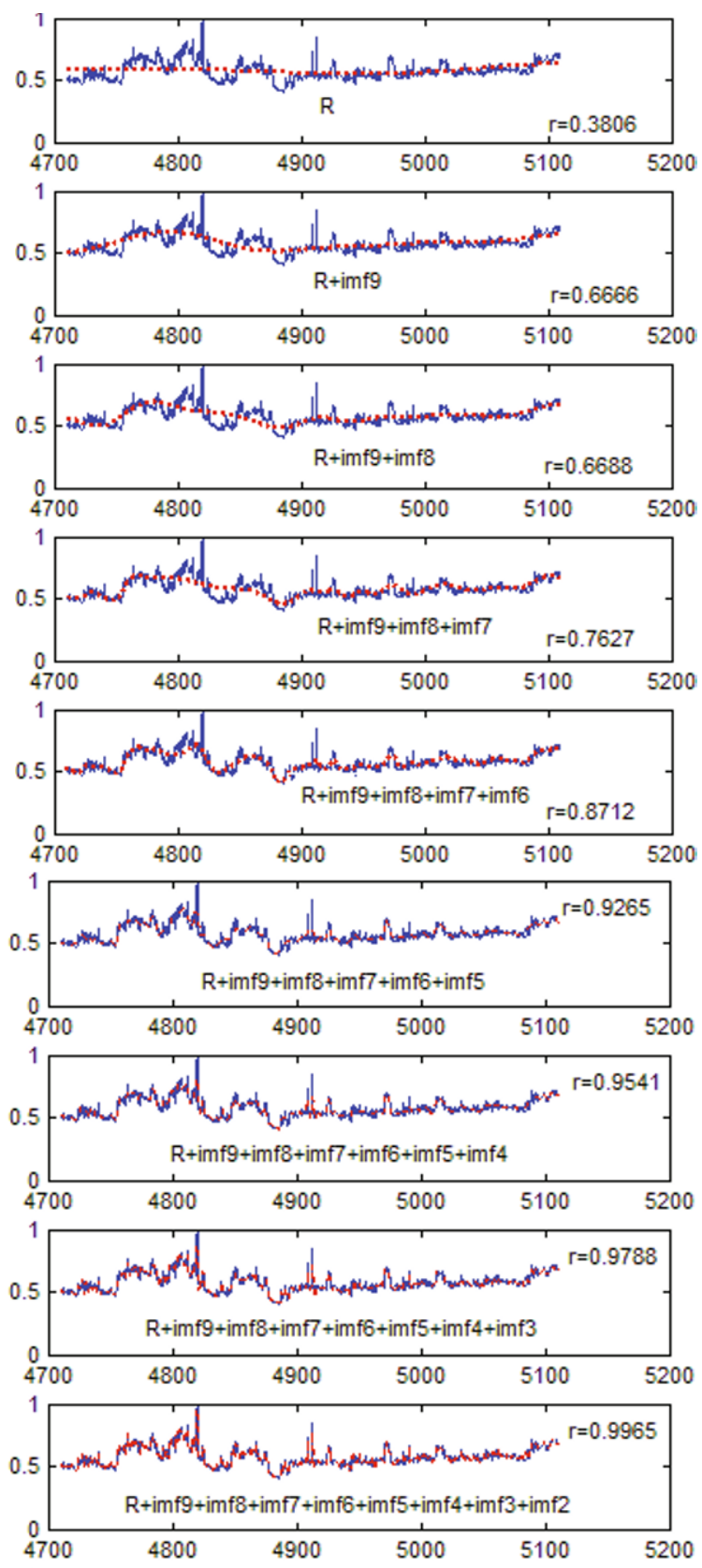

Fig. 3. Reconstruction of GR_SP components by using IMF (GS_SP - solid line, reproduced sequence - broken line, $r$ - correlation coefficient of reproduced sequence with GR_SP). 
Cycle grade was a division on stratigraphic characteristics at different scales. The high frequency component in every component of EMD showed that it had the details. These details included useful information and also contained noise interference. Sedimentary cycle was a relatively stable process; the middle and low frequencies in the signals deserved more attention. Those high frequency signals were used but with caution.

In Table 2 the set $r_{i}(i=1-9)$ of correlation coefficients of GR_SP and its EMD functions is presented. Correlation coefficients of original signal and its mode functions $\left(\mathrm{IMF}_{i}\right)$ were calculated by using the correlation function corrcoef () provided by MATLAB (Table 2 ).

The minimal value in Table 2 is $r_{1}=0.0962$ which meant that IMF 1 has the poorest correlation with GR_SP. That result showed that IMF1 contained a lot of noise components with high frequency. On the contrary, $r_{9}$ is the maximum value showing the best correlation. That low-frequency appears in IMF9 indicates that it contains less noise. Furthermore, EMD functions of IMF5, IMF6, and IMF7 also had high correlation with GR_SP. These results are same as the conclusion drawn from the above-mentioned EMD diagram in Fig. 3.

In the same way the mutual relationships were calculated between IMFs and the correlation coefficients were presented in Table 3.

The correlation coefficients values of intrinsic mode functions were close to zero, so there was a conclusion that they had no linear correlation between them. Yet, they could have high local correlation of some segments.

Table 2

Correlation coefficients of EMD functions of $\mathrm{IMF}_{i}$ and GR_SP

\begin{tabular}{|c|c|c|c|c|c|c|c|c|}
\hline$r_{1}$ & $r_{2}$ & $r_{3}$ & $r_{4}$ & $r_{5}$ & $r_{6}$ & $r_{7}$ & $r_{8}$ & $r_{9}$ \\
\hline 0.0962 & 0.1656 & 0.2463 & 0.2303 & 0.4167 & 0.3698 & 0.3333 & 0.1858 & 0.5255 \\
\hline
\end{tabular}

Table 3

Correlation coefficient value of intrinsic mode functions $\left(\mathrm{IMF}_{i}\right)$ under EMD

\begin{tabular}{|l|c|c|c|c|r|r|r|r|}
\hline & IMF2 & IMF3 & IMF4 & \multicolumn{1}{c|}{ IMF5 } & \multicolumn{1}{c|}{ IMF6 } & \multicolumn{1}{c|}{ IMF7 } & \multicolumn{1}{c|}{ IMF8 } & \multicolumn{1}{c|}{ IMF9 } \\
\hline IMF1 & 0.086813 & -0.00652 & -0.01767 & -0.00617 & -0.0059 & 0.00204 & 0.000747 & 0.00209 \\
IMF2 & & -0.01602 & -0.02439 & -0.04389 & 0.033146 & 0.039231 & -0.04593 & -0.00704 \\
IMF3 & & & 0.072093 & 0.016625 & -0.04814 & -0.00913 & 0.025364 & 0.03056 \\
IMF4 & & & & 0.046212 & -0.00958 & -0.01612 & -0.01182 & -0.01455 \\
IMF5 & & & & & 0.097756 & 0.051052 & -0.11136 & 0.106921 \\
IMF6 & & & & & & 0.118184 & -0.1617 & -0.07389 \\
IMF7 & & & & & & & -0.00862 & -0.04254 \\
IMF8 & & & & & & & & -0.09877 \\
\hline
\end{tabular}




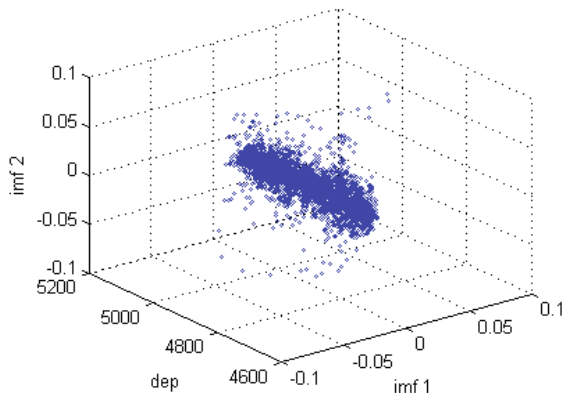

(a)

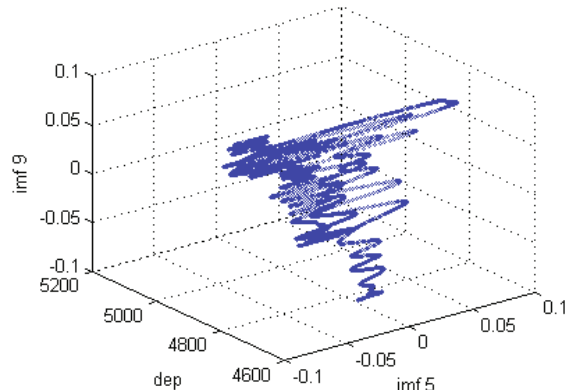

(c)

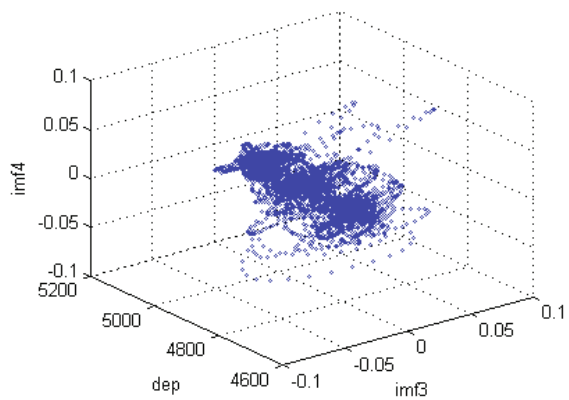

(b)

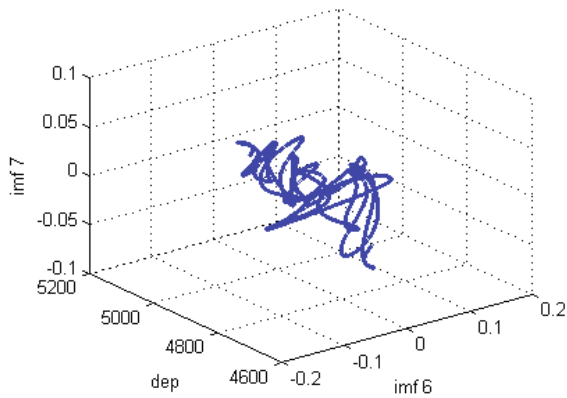

(d)

Fig. 4. IMF components scatter plot.

IMF1-IMF2 and IMF3-IMF4 with less correlation to GS_SP were selected as one group and MF5-IMF9 and IMF6-IMF7 with better correlation to GS_SP were selected as the other group, the scatter diagrams were made in depth domain (Fig. 4). The scatter diagram for the combination of IMF1 and IMF2 as well as IMF3 and IMF4 were disorderly. They all had poor correlation with GR_SP. Furthermore, the correlation coefficients in Table 3 were comparatively low. So, the combination of IMF5 and IMF9 as well as IMF6 and IMF7 not only revealed better correlation, they also had higher correlation coefficients values. Their scatter diagrams were smooth curves, which showed their rules.

In order to further the explanation of the discussed problem, the periodic functions and non-periodic functions with lower correlation coefficients as well as random sequences were selected, respectively (Figs. 5a-c) (Bogle et al. 1994, Hoste and Zirbel 2006).

In the first plot in each group in Figs. 5a-c there are presented two original signals; $r$ is the correlation coefficient. In the second plot of each group in Figs. 5a-c there is the overlapped signal of those two original signals given in the first figure. In the third plot there is the scatter diagram of those two original signals. 

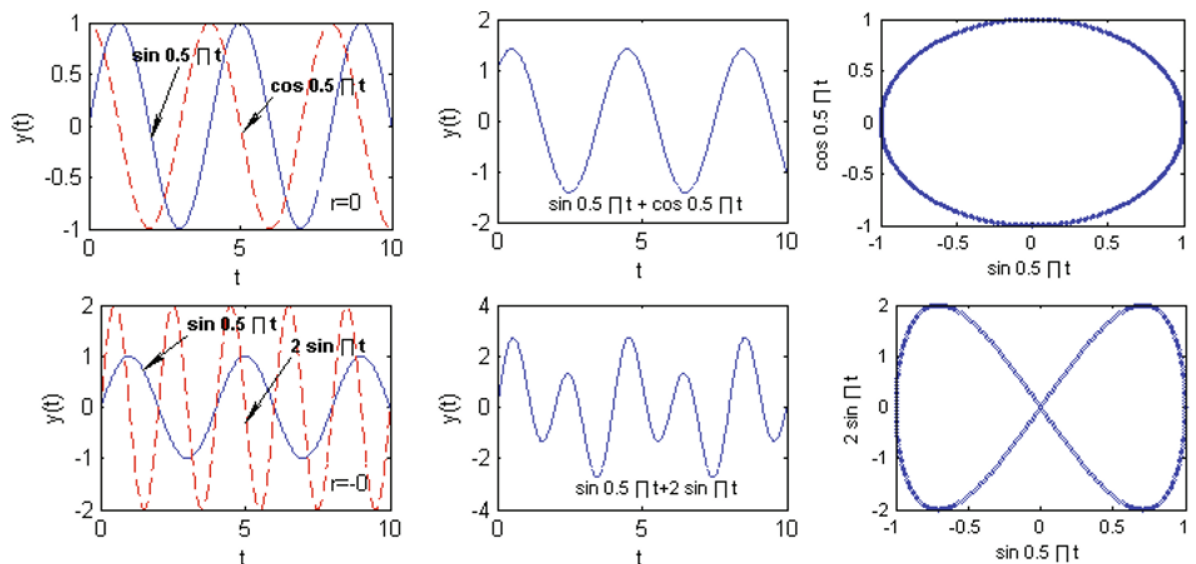

Fig. 5a. Scatter diagram of two groups of non-linear correlation periodic functions.
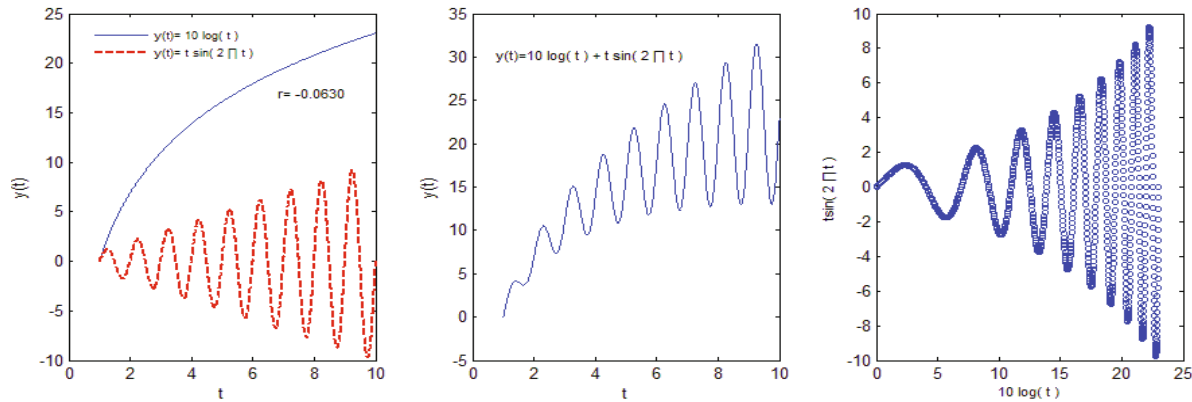

Fig. 5b. Scatter diagram of non-linear correlation and non-periodic functions.
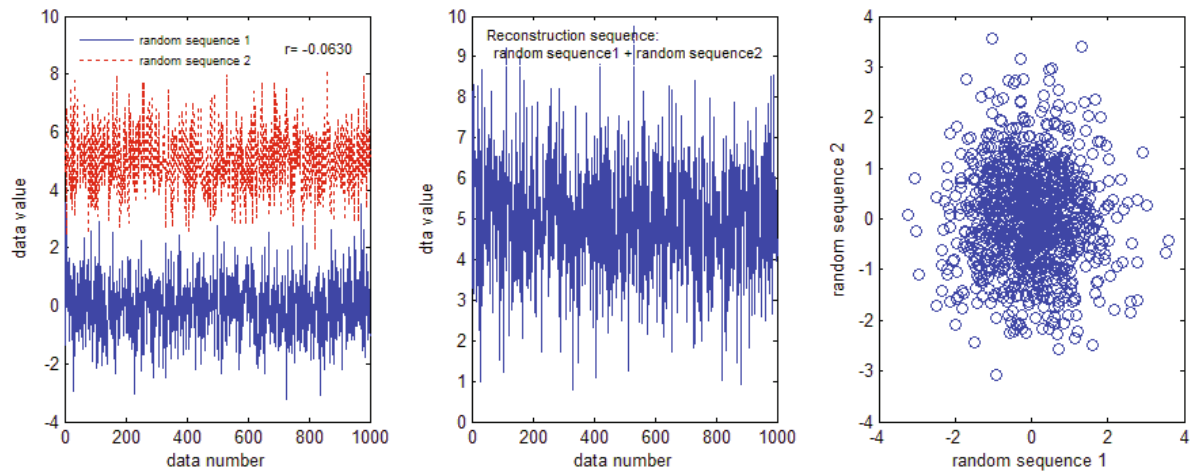

Fig. 5c. Scatter diagram of random sequences for non-linear correlation.

In Fig. 5a there is an example of periodic function adopted to make the scatter diagram of functions with same frequency and different phases as well as the functions with same phase and different frequency. The curves of 
two periodic functions of non-linear correlation still had periodicity after overlapping. Then, their scatter diagrams were stable closed curves. In Fig. $5 \mathrm{~b}$ the presented examples belong to non-periodic functions of nonlinear correlation. After combination they are still non-periodic functions and their scatter diagrams are the curves that will never close. In Fig. 5c there are random sequences of non-linear correlation; when they are combined, they still belong to non-periodic random sequence and their scatter diagrams are disordered.
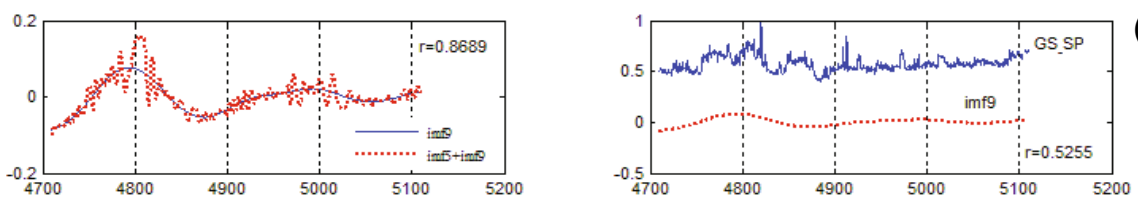

(a)
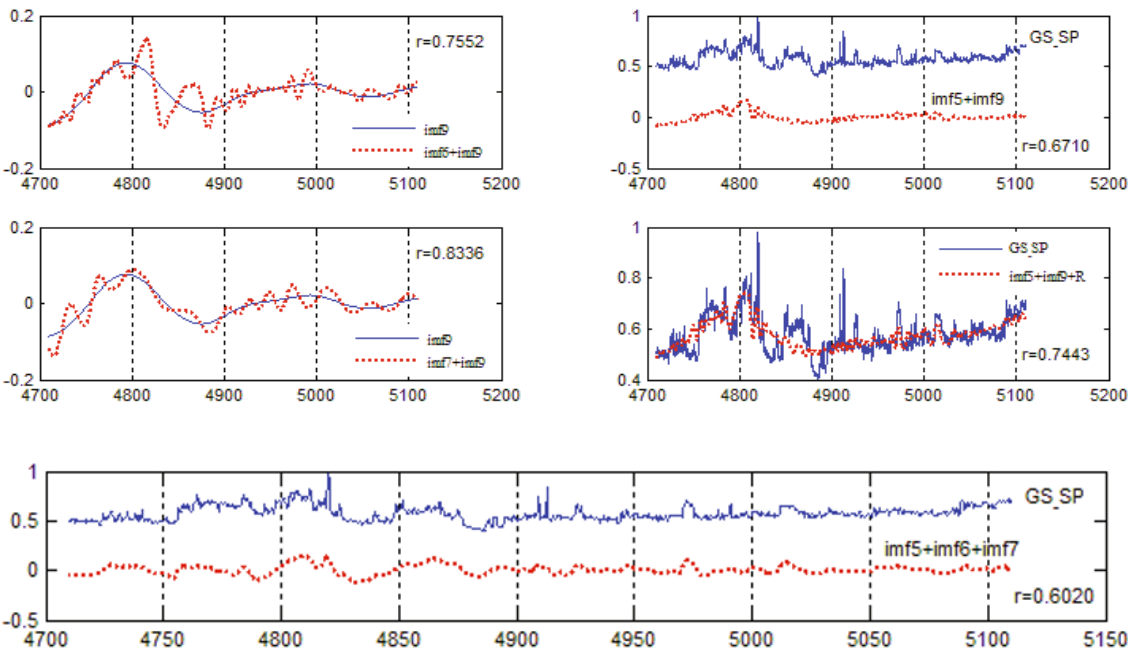

(b)
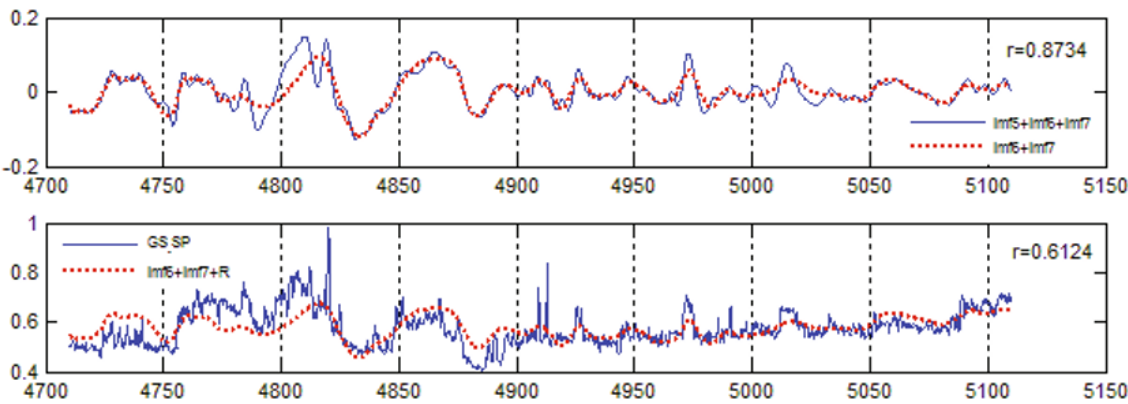

Fig. 6. Comparison of low frequency signals combination: (a) comparison with the related signal of IMF5+IMF9, and (b) comparison with the related signal of IMF6+IMF7; $\mathrm{r}$ - correlation coefficient. 
The scatter diagrams of IMF5 and IMF9, IMF6 and IMF7 are smooth curves (Fig. 4c and d). Figure $4 \mathrm{c}$ has the correlative characteristics of piecewise curve and they have no obvious closed diagram while Fig. $4 \mathrm{~d}$ has many staged closed diagrams in the scatter diagram. According to sedimentology, the stratum in different periods may have different sedimentation rules while the stratum in the same period may have similar characteristics; these conform to the rules reflected by scatter diagram. Accordingly, the combination of IMF5 and IMF9 is suitable for researching sedimentation rules of the stratum in middle or long-term and the combination of IMF6 and IMF7 is suitable for researching the short term sedimentation rules of the stratum.

In Fig. 6, from the correlation of combined signals we can further prove that the overlapped sequences of IMF5 and IMF9 as well as IMF6 and IMF7 can best reveal the rules of long-term cycles and the short-term cycle.

Besides, the combined sequence of IMF5+IMF9 has higher correlativity with IMF9 of low frequency component, which obviously improves the correlativity of IMF9 and GS_SP due to participation of IMF5. Similarly, IMF6+IMF7 not only well reflect the rule of IMF5+IMF6+IMF7, but also have good correlation with GS_SP.

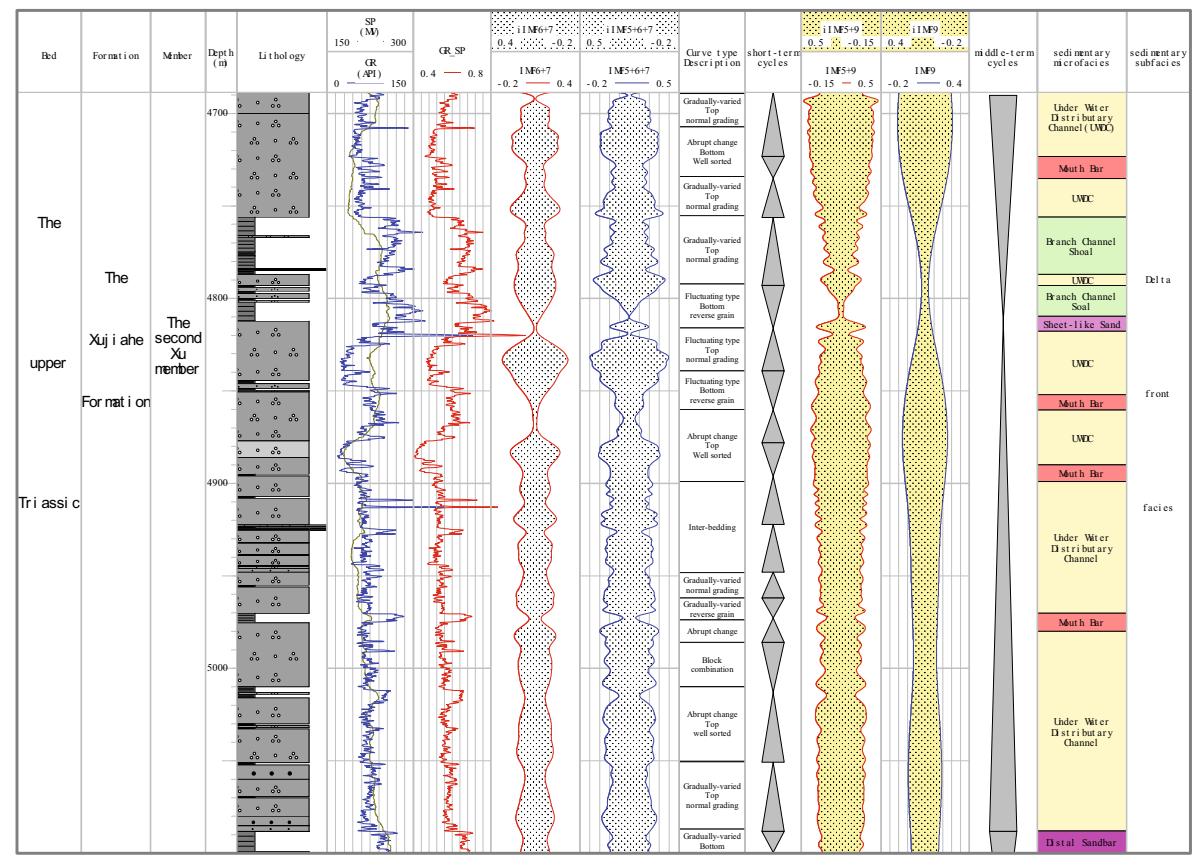

Fig. 7. Plots illustrating identification of short and middle-term cycle sequence stratum of $\mathrm{X}$ well using EMD method. 
Further analysis showed that combining the decomposition signals under EMD resulted in strengthening of intrinsic information reflecting stratum characteristics and declined the influence of noise interference. The compared result defined that in the all combined signals of IMFs, only those which have good correlation index between each other and also have good correlation index with the original signal can better reveal the cycle characteristics of stratum. In Fig. 7 the results of EDM analysis are presented.

GR_SP was a new combined parameter between natural gamma ray and spontaneous potential. IMFs were the results of empirical mode decomposition (EMD) of GR_SP. The IMF6+7 denoted IMF6 plus IMF7 and IMF5+9 denoted IMF5 plus IMF9. Both iIMF6+7 and iIMF5+9 represented their mirror image, respectively. Therefore, IMF5+6+7 and iIMF $5+6+7$, IMF9 and iIMF9 have the same rule as before.

These combined curves not only contained changes of the lithology, but also revealed the trend in cycle of sequence stratum. In order to identify short and middle-term cycle sequence stratum effectively, we should refer to Table 1 (discussed in Section 3.2.2); we analyzed the classification of these curves and found out the normal graded bed sequence and reverse grading.

\section{DISCUSSION}

$\mathrm{X}$ well is located at Xiaoquan-Fenggu Structure Belt, middle section of West Sichuan Depression, Sichuan Basin. In Fig. 7 there are shown the characteristics of base level cycle of the stratum, the second section of Xujia River group. Its lithology includes mainly fine and middle grained sandstone and shale, belonging to delta front sub-facies. The combined signals with different frequencies were analyzed after EMD of curve characteristics according to Table 1. Short-term or long-term cycle characteristics were obviously identified and the sedimentary micro-facies were presented as follows:

Under water distributary channel: its lithology includes grey finegrained to middle coarse particle sandstone. It represents the characteristics of cycle of positive graded bedding. It belongs to cross bedding, inclined bedding, and plot bedding. The scour and fill structure can be seen.

Mouth bar: its lithology chiefly is well sorted silt or fine sand and filled with cross-bedded sandstone, which has the characteristics of reverse grain size order cyclic-sequence.

Branch channel shoal: its lithology chiefly includes clay and contains less fine sand or silt. It is horizontally bedding.

Sheet-like sand: its lithology is separated into sorted fine sand or silt. It is cross-bedding and has the characteristics of reverse grain size order cyclicsequence. 
Distal sandbar: it is located at the farther part before estuary dam and its lithology mainly is silt and filled with cross-bedded sandstone, which has the characteristics of reverse grain size order cyclic-sequence.

\section{CONCLUSIONS}

口 Logging indices reflected the physical property characteristics of stratum's lithology, formation, sequence, and reservoir rocks. Sensitive indices were selected to improve the accuracy of logging interpretation.

- Spontaneous potential and natural gamma ray were the sensitive indices reflecting geological formation and lithology. The mathematical methods were adopted for selecting new parameters and combining them. The new parameters strengthened the intrinsic characteristics and improved the results of partition.

- EMD is an innovative time-frequency analysis method; not only does it guarantee the inherent characteristics in non-linear and non-stationary processes after the decomposition of signal, but also avoids the limitation of windowed Fourier Transform being constrained by time window and wavelet transform influenced by basis function. It can accurately describe the time-varying characteristics of signals. In fact, every $\mathrm{IMF}_{i}$ may has a physical meaning (i.e., it may be related to geological phenomena.), and, as already highlighted, an important issue in practical application is how to judge the existence of this meaning in each $\mathrm{IMF}_{i}$. This calls for further study and discussion.

口 EMD method was adopted for various combined logging indices; a discussion on relativity of mode functions and a search for the valued combinations are presented. Creating $\mathrm{IMF}_{i}$ combined signal helps to obtain abnormal dots (e.g., mutations in different sedimentary layer interface) and better identify the cycle of stratigraphic base level.

\section{References}

Akansu, A.N., W.A. Serdijn, and I.W. Selesnick (2010), Emerging applications of wavelets: A review, Phys. Comm. 3, 1, 1-18, DOI: 10.1016/j.phycom.2009. 07.001 .

Bogle, M.G.V., J.E. Hearst, V.F.R. Jones, and L. Stoilov (1994), Lissajous knots, J. Knot Theor. Ramif. 3, 2, 121-140, DOI: 10.1142/S0218216594000095.

Bowman, D.C., and J.M. Lees (2013), The Hilbert-Huang transform: A high resolution spectral method for nonlinear and nonstationary time series, Seismol. Res. Lett. 84, 6, 1074-1080, DOI: 10.1785/0220130025. 
de Lima, E.R., A.O. Andrade, J.L. Pons, P. Kyberd, and S.J. Nasuto (2006), Empirical mode decomposition: a novel technique for the study of tremor time series, Med. Biol. Eng. Comput. 44, 7, 569-582, DOI: 10.1007/s11517-0060065-x.

Flandrin, P., G. Rilling, and P. Gonçalvés (2004), Empirical mode decomposition as a filter bank, IEEE Signal Process. Lett. 11, 2, 112-114, DOI: 10.1109/ LSP.2003.821662.

Hoste, J., and L. Zirbel (2006), Lissajous knots and knots with Lissajous projections, arXiv: math/0605632 [math.GT], 1-17.

Huang, N.E., and Z. Wu (2008), A review on Hilbert-Huang transform: Method and its applications to geophysical studies, Rev. Geophys. 46, 2, RG2006, DOI: 10.1029/2007RG000228.

Huang, N.E., Z. Shen, S.R. Long, M.C. Wu, H.H. Shih, Q. Zheng, N.C. Yen, C.C. Tung, and H.H. Liu (1998), The empirical mode decomposition and the Hilbert spectrum for nonlinear and non-stationary time series analysis, Proc. R. Soc. Lond. A. 454, 1971, 903-995, DOI: 10.1098/rspa.1998.0193.

Huang, N.E., Z. Shen, and S.R. Long (1999), A new view of nonlinear water waves: the Hilbert spectrum, Ann. Rev. Fluid Mech. 31, 417-457, DOI: 10.1146/ annurev.fluid.31.1.417.

Li, Y.-J., X.-Y. Hu, and Z.-X. He (2010), Application situation and prospects of Hilbert-Huang transform in geophysics, Chin. J. Eng. Geophys. 7, 5, 537-543, (in Chinese).

Serra, O., and H.T. Abbott (1982), The contribution of logging data to sedimentology and stratigraphy, Soc. Petrol. Eng. J. 22, 1, 117-131, DOI: 10.2118/ 9270-PA.

van Wagoner, J.C., R.M. Mitchum, K.M. Campion, and V.D. Rahmanian (1990), Siliciclastic Sequence Stratigraphy in Well Logs, Cores, and Outcrops: Concepts for High-Resolution Correlation of Time and Facies, AAPG Methods in Exploration Series, No. 7, The American Association of Petroleum Geologists, Tulsa, USA.

Wawrzyniak, K. (2010), Application of time-frequency transforms to processing of full waveforms from acoustic logs, Acta Geophys. 58, 1, 49-82, DOI: 10.2478/s11600-009-0043-4.

Wu, Z., and N.E. Huang (2004), A study of the characteristics of white noise using the empirical mode decomposition method, Proc. R. Soc. Lond. A 460, 2046, 1597-1611, DOI: 10.1098/rspa.2003.1221.

Zhang, T., and C.-Y. Nie (2011), Information extraction of acoustic logging for oil layers based on EMD time-frequency analysis, J. Changchun Univ. 21, 8, 15-18 (in Chinese).

Zheng, R.-C., S.-M. Yin, and J. Peng (2000), Sedimentary dynamic analysis of sequence structure and stacking pattern of base-level cycle, Acta Sediment. Sin. 18, 3, 369-375 (in Chinese). 
Zheng, R.-C., J. Peng, and C.-R. Wu (2001), Grade division of base-level cycles of terrigenous basin and its implications, Acta Sediment. Sin. 19, 2, 249-255 (in Chinese).

Zheng, T.-X., and L.-H. Yang (2007), Discussion and improvement on empirical mode decomposition algorithm, Acta Scient. Nat. Univ. Sunyatseni 46, 1, 1-6 (in Chinese).

Received 28 March 2014 Received in revised form 6 October 2014

Accepted 15 October 2014 